\title{
ECR Heating in a Helicon Device
}

\author{
Jacob Zalach, Olaf Grulke and Thomas Klinger
}

Max-Planck Institute for Plasma Physics, EURATOM Association, Greifswald, Germany

\begin{abstract}
This paper presents a microwave electron cyclotron resonance heating system for the linear magnetized VINETA helicon device. Thereby, a relatively cold helicon wave generated plasma is heated by resonant electron cyclotron waves. The magnetic field configuration is adjusted such that resonant energy transfer occurs locally to heat the plasma electrons. Electron temperatures of $12 \mathrm{eV}$ are obtained for an Argon plasma at a pressure of $0.02 \mathrm{~Pa}$. The absorption of the microwave across the resonance layer is measured with capacitive probes. Plasma parameters are characterized using compensated Langmuir probes. Additionally, a $160 \mathrm{GHz}$ microwave interferometer is used to measure the line integrated plasma density. The evolution of these quantities for different microwave powers is compared to power balance computations, which indicate that the applied microwave power is dissipated by the ionization collisions; after saturation of ionization processes the power is transferred into electron kinetic energy.
\end{abstract}

Keywords: ECR heating, linear device

PACS: $52.50 .-\mathrm{b}$

\section{INTRODUCTION}

Helicon plasma sources have attracted great attention due to the very efficient plasma production at moderate rf power $[1,2]$. With only few $\mathrm{kW}$ rf power plasma densities in the range of $n_{e} \sim 10^{19} \mathrm{~m}^{-3}$ are achieved $[3,4,5]$. Therefore helicon plasmas are often used in basic research and have attracted much interest for technical applications [6]. Nevertheless, the electron temperatures in helicon plasmas are generally low (in the range of a few eV). The combination of high plasma density and low electron temperature leads to a highly collisional plasma, in which Coulomb collisions play a dominant role in plasma dynamical phenomena [4]. This work presents an electroncyclotron-resonance (ECR) heating setup for a helicon plasma produced in the linear VINETA device. The final goal is to increase the plasma temperature and thereby to decrease the plasma collisionality. The paper is structured as follows: In Sec. 2 we describe the experimental setup and the diagnostic tools used. Measurements of microwave absorption and plasma parameters for a pure ECR and a combined ECR-helicon discharge are presented in Sec. 3. Finally the results are discussed in Sec. 4.

\section{EXPERIMENTAL SETUP AND DIAGNOSTIC}

The ECR setup is part of the VINETA device $[4,7,8,9,10]$ which is a cylindrical, linearly magnetized plasma experiment with a total length of $L=4.5 \mathrm{~m}$ and $D=0.4 \mathrm{~m}$ diameter. Fig. 1 shows a schematic drawing of VINETA indicating the position of the helicon source and the ECR heating setup.

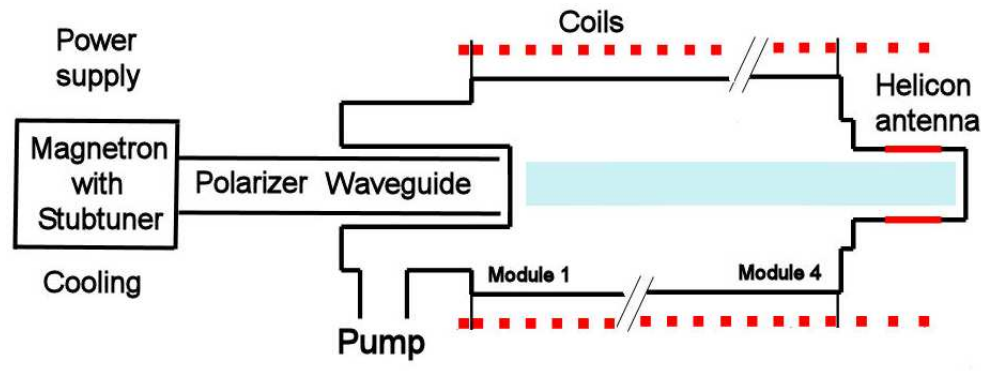

FIGURE 1. Schematic of the VINETA device with the helicon source and the ECR setup at opposite ends. 
At the one end of the device a conventional helicon source is installed for plasma production. The helical $m=1$ antenna is placed around a cylindrical glass vacuum extension $(0.5 \mathrm{~m}$ length, $0.1 \mathrm{~m}$ diameter $)$ and is connected to an $\mathrm{rf}$ source via a capacitive matching unit. The source is driven at a frequency of $f_{\mathrm{rf}}=13.56 \mathrm{MHz}$ with a maximum power of $P_{\mathrm{rf}, \max } \leq 5 \mathrm{~kW}$. A detailed description of the helicon setup and its operation is given in Ref. [7].

At the opposite end to the VINETA device the ECR heating setup is installed. Microwaves were previously used on linear magnetized devices as plasma sources $[11,12,13,14]$. The goal is a direct coupling of the microwave electric field to the electron gyro motion which allows resonant energy transfer to the plasma electrons. An overview of the principle is given in [15]. Our system consists of a standard industrial microwave generator (Mügge Type MS010KE$110 \mathrm{DE}$ ) with a maximum continuous power output of $P_{\mathrm{mw}} \leq 10 \mathrm{~kW}$ at a frequency of $f_{\mathrm{ECR}}=2.45 \mathrm{GHz}$. The linearly polarized microwave $\left(H_{01}-\right.$ mode $)$ is first transferred to a $H_{11}$ - mode and then passes a polarizer, which transforms it to a right-handed polarized wave (R-wave). The microwave antenna, which is a cut waveguide, is placed directly in the plasma center and the R-wave is launched along an inhomogenius magnetic field. The magnetic field gradient and thereby the resonant absorption layer is produced by non-equidistant spaced magnetic field coils placed around the steel vessel. For resonant absorption the magnetic field has to be adjusted such, that the electron cyclotron frequency is equal to the microwave frequency $\omega_{\mathrm{ecr}}=\omega_{\mathrm{o}}$. The required magnetic field strength is $B_{\text {res }}=87.5 \mathrm{mT}$. The magnetic field inhomogenity is restricted to a region close to the microwave antenna $(\approx 1 / 4 L)$, while the rest of VINETA is homogeneously magnetized. The microwave antenna is vacuum sealed with a quartz window. Any mismatching of the microwave is compensated using a 3-stub-tuner system. Typical reflected power levels are a few tens of Watt.

Passively compensated Langmuir probes $[16,17]$ are used to measure the electron temperature and density in the plasma center by standard evaluation of probe characteristics. A capacitive probe [18] is used in the vacuum chamber center at an axial distance of $0.9 \mathrm{~m}$ from the microwave antenna in order to measure the electric field of the microwaves. Its power spectrum is analyzed and the comparison between the magnitudes of the microwave peak at $\omega_{\mathrm{ECR}}$ with and without plasma gives an estimate of the microwave absorption across the resonant magnetic field layer. As an independent method a $160 \mathrm{GHz}$ microwave interferometer measures the line integrated plasma density $\left(\int n_{e} \mathrm{~d} r\right)$, which is compared with the radially integrated ion saturation current profiles $\left(\int n_{e} \sqrt{T_{e}} \mathrm{~d} r\right)$. Any deviation from a linear relationship between this two independently measured quantities can be directly attributed to the electron temperature profile.

\section{MEASUREMENTS}

All measurements presented here were done in Argon at a pressure of $0.02 \mathrm{~Pa}$ with an axial magnetic field contour as shown in Fig. 2(a). Absorption properties were investigated with a capacitive probe in a pure ECR discharge. The microwave signal is strongly reduced or completely disappeares as soon as the absorption layer is reached. A typical measurement is shown in Fig. 2(b). The measured signal drops from values of $-5 \mathrm{~dB}$ (without plasma) to roughly $-50 \mathrm{~dB}$ (with plasma). Furthermore the width of the peak is strongly reduced by the wave-plasma interaction. This behaviour is qualitatively the same for all microwave powers. The evolution of the radial ion saturation current profiles
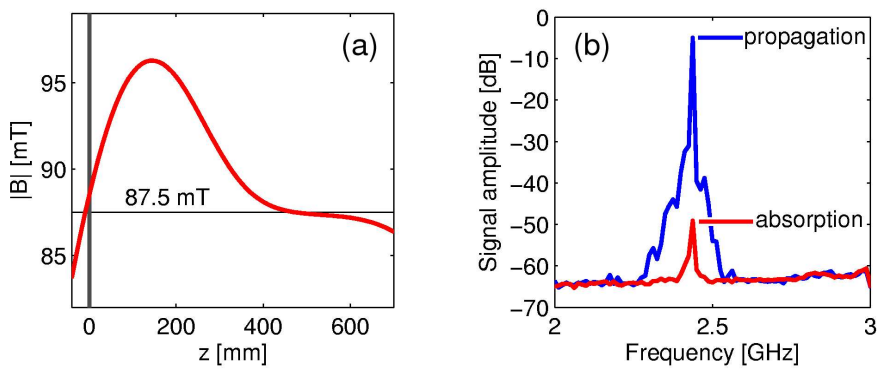

FIGURE 2. Left: Central axial magnetic field strength. Right: Spectrum of the capacitive probe signals for the two cases of absorption and free wave propagation without plasma.

and of the electron temperature with increasing microwave power is shown in Fig. 3 and Fig. 4. The profiles were measured for a pure ECR discharge [Fig. 3(a)] and a combined ECR discharge with additional $2 \mathrm{~kW}$ rf power from the helicon source [Fig. 3(b)] (combined discharge). The comparison of the ion saturation current profiles show that the height of the profile increases with the microwave power for both the pure ECR and the combined discharge. Furthermore, the profiles for comparable microwave power are wider for the combined discharge. The shoulder- 

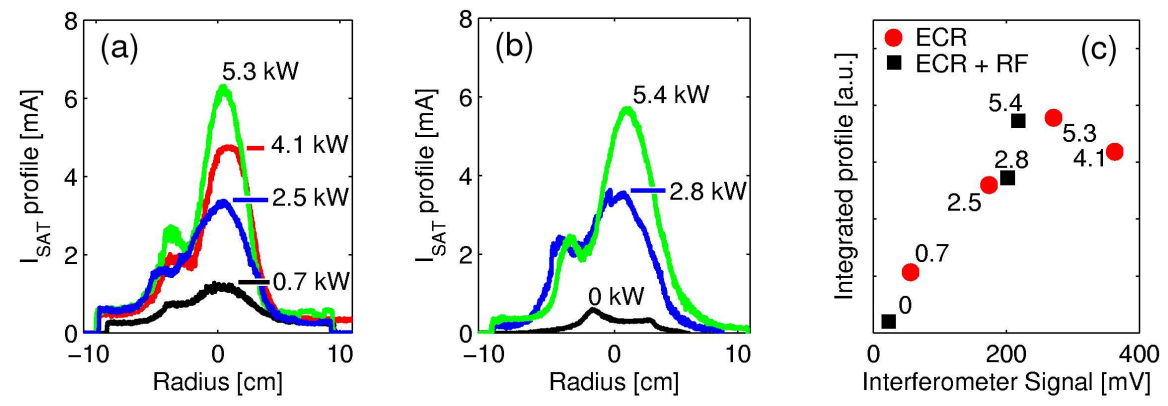

FIGURE 3. Ion saturation current profiles for a pure ECR discharge (left) and a combined discharge with ECR and $2 \mathrm{~kW}$ rf power (middle).The right diagram shows a comparison between line integrated density measured with the microwave interferometer $\left(\sim n_{e}\right)$ and radially integrated ion saturation current profiles obtained by Langmuir probes $\left(\sim n_{e} \sqrt{T_{e}}\right)$. The nonlinear relationship is caused by a temperature difference. Every marker represents averaged values of several measurements, the applied microwave power is given in $\mathrm{kW}$.

like structure which occurs in all ECR plasma profiles supposedly originates from a misalignment of the microwave antenna. The comparison of the microwave interferometer measured line integrated density with the radially integrated ion saturation current profiles for the pure ECR and combined discharge is shown in Fig. 3(c). For both discharge types the value of the integrated ion saturation current profile increases monotonically with applied microwave power. The interferometer signal increases for the pure ECR discharge with microwave power of $4.1 \mathrm{~kW}$ but decreases for higher powers. The linear increase in the case of the combined discharge becomes slightly stronger at microwave powers above $2.8 \mathrm{~kW}$. The central temperature dependence on the applied microwave power [Fig. 4(a)] was obtained with two probes separated by $1.2 \mathrm{~m}$ from each other in a pure ECR discharge. The temperature values of probe 2 are higher than those of probe 1 which has a higher distance to the antenna. Both probes show a plateau like behavior up to microwave power of $3-4 \mathrm{~kW}$. Higher power values results in a temperature increase up to about $12 \mathrm{eV}$ at roughly 6 $\mathrm{kW}$. By applying additional $2 \mathrm{~kW} \mathrm{rf}$ power the temperature changes as shown in Fig. 4(b) The temperature values are significantly lower than in the case of the pure ECR discharge.

\section{DISCUSSION}

The measurements with the capacitive probes show a strong damping of the microwave signal as soon as the absorption layer is reached which means that resonant absorption actually takes place. The ion saturation current profiles and the central temperature develop with increasing microwave power as expected. We note that ion saturation current is dependend on plasma density and temperature
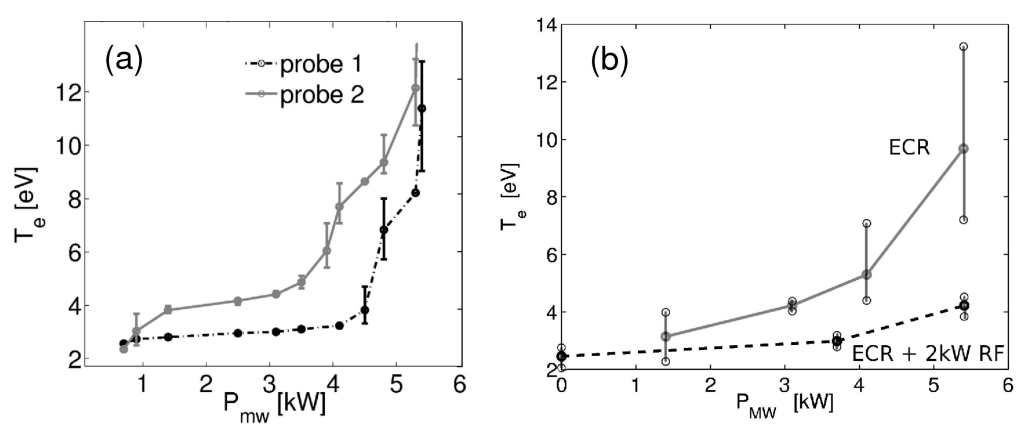

FIGURE 4. Electron temperature taken in the plasma center: left) at two different axial positions for a pure ECR discharge (probe distance $1.2 \mathrm{~m}$ ) and right) comparison of a pure ECR and a combined discharge with ECR and $2 \mathrm{~kW}$ rf power. 


$$
I_{\text {sat }}(r)=k e A_{\text {probe }} n_{i}(r) e \sqrt{\frac{T_{e}(r)}{M_{i}}}
$$

where $M_{i}$ is the ion mass, $A_{\text {probe }}$ the projection of the effective area of the collecting probe in the direction of the magnetic field and $k=0.49$ is a constant which includes the influence of the magnetic field [19]. The comparison of the radially integrated ion saturaton current profile with the line integrated density as measured with the interferometer [Fig. 3(c)] indicates, that at microwave power levels above $4 \mathrm{~kW}$ the temperature increases. This finding is supported by probe measurements (Fig. 4), where an electron temperature increase is observed only for microvave power levels $P_{E C R} \geq 4 \mathrm{~kW}$. This behavior can be understood with the help of power and particle balance computations [20]. In the computation the main sources and sinks of particles and energy - ionization, recombination, excitation of atoms, radiation and elastic electron-ion collisions - are considered as well as the open ended linear geometry of the VINETA. They result in a relationship between $n_{e}$ and $T_{e}$ where the density decreases with increasing electron temperature. It turns out that for the ECR discharge the applied microwave power is first absorbed by ionization processes which increase the density, as observed in Fig. 3(c). At a particular power level the ionization mechanism saturates and the remaining power heats the plasma electrons and the density decreases. For the pure ECR discharge this point is reached at $4.1 \mathrm{~kW}$. This interpretation is supported by the temperature measurements shown in Fig. 4(a). The values for the combined discharge show a different behavior. The interferometer data show that the linear increase above $2.8 \mathrm{~kW}$ becomes slightly stronger. This behavior corresponds with the temperature measurements shown in Fig. 4(b) which also show a slight increase.

\section{SUMMARY}

The present setup is suitable to obtain a ECR heated plasma with significant higher electron temperatures than in the case of a pure helicon discharge at plasma densities in the range of $10^{17}-10^{18} \mathrm{~m}^{-3}$. Resonant absorption takes place and the ion saturation current profiles as well as the microwave interferometer data show a relationship which can be understood with the help of power balance computations where first the absorbed power is mainly used by the ionization mechanism which eventually saturate. Beyond that point power increase leads to an increase of electron temperature. This explanation is supported by temperature measurements which develop with the applied microwave power as expected.

\section{REFERENCES}

1. F. F. Chen, and R. W. Boswell, IEEE Trans. Plasma Sci. 25, 1245-1257 (1997).

2. R. W. Boswell, and F. F. Chen, IEEE Trans. Plasma Sci. 25, 1229-1244 (1997).

3. F. F. Chen, J. Vac. Sci. Technol. A 10, 1389 (1992).

4. C. Schröder, O. Grulke, T. Klinger, and V. Naulin, Phys. Plasmas 11, 4249-4253 (2004).

5. F. F. Chen, I. D. Sudit, and M. Light, Plasma Sources Sci. Technol. 5, 173-180 (1995).

6. I. Tempermeister, J. Vac. Sci. Technol. B 12, 2310 (1994).

7. C. M. Franck, O. Grulke, and T. Klinger, Phys. Plasmas 10, 323-325 (2002).

8. C. M. Franck, O. Grulke, and T. Klinger, Phys. Plasmas 9, 3254-3258 (2002).

9. C. M. Franck, O. Grulke, T. Klinger, A. Stark, E. E. Scime, and G. Bonhomme, Plasma Sources Sci. Technol. 14, 226-235 (2005).

10. O. Grulke, C. M. Franck, T. Klinger, C. Schröder, A. Stark, T. Windisch, and J. Zalach, Contrib. Plasma Phys. 45, 385-395 (2005).

11. V. P. Bhatnagar, G. VanOost, A. M. Meissiaen, and P. E. Vandenplas, Plasma Physics 18, 535-540 (1975).

12. D. Hemmers, M. David, H. Kempkens, and J. Uhlenbusch, J. Phys. D: Appl. Phys. 31, 2155-2164 (1998).

13. V. Yadav, and D. Bora, Plasma Sources Sci. Technol. 13, 231-236 (2004).

14. P. Reinke, S. Schelz, W. Jacob, and W. Möller, J. Vac. Sci. Technol. A 10, 434-438 (1992).

15. M. A. Lieberman, and A. J. Lichtenberg, Principles of plasma discharges and materials processing, Wiley-Interscience, 1994.

16. I. D. Sudit, and F. F. Chen, Plasma Sources Sci. Technol. 3, 162-168 (1994).

17. U. Flender, B. H. Nguyen-Thi, K.Wiesemann, N.A.Khromov, and N.B.Kolokolov, Plasma Sources Sci. Technol. 5, 61-69 (1995).

18. S. E. Savas, and K. G. Donohoe, Rev. Sci. Instrum. 60, 3391-3395 (1989).

19. D. Batani, S. Alba, P. Lombardi, and A. Galassi, Rev. Sci. Instrum. 68, 4043-4050 (1997).

20. C. Lechte, J. Stöber, and U. Stroth, Phys. Plasmas 9, 2839-2846 (2002). 